\title{
Phase II trial of biweekly docetaxel, cisplatin, and 5-fluorouracil chemotherapy for advanced esophageal squamous cell carcinoma
}

\author{
Yoshihiro Tanaka $^{1} \cdot$ Kazuhiro Yoshida $^{1} \cdot$ Atsuko Yamada $^{1} \cdot$ Toshiyuki Tanahashi $^{1} \cdot$ \\ Naoki Okumura $^{1} \cdot$ Nobuhisa Matsuhashi $^{1} \cdot$ Kazuya Yamaguchi $^{1}$. \\ Tatsuhiko Miyazaki ${ }^{2}$
}

Received: 9 November 2015 / Accepted: 4 February 2016 / Published online: 20 February 2016

(c) The Author(s) 2016. This article is published with open access at Springerlink.com

\begin{abstract}
The prognosis of esophageal cancer patients is still unsatisfactory. Although a docetaxel, cisplatin, and 5 -Fu (DCF) regimen has been reported, it is often difficult to accomplish because of severe toxicity. Therefore, we developed a new biweekly DCF (Bi-DCF) regimen and previously reported the recommended dose in a phase I dose-escalation study. We then performed a phase II study of Bi-DCF for advanced esophageal squamous cell carcinoma (SCC). Patients with clinical stage II/III were eligible. Patients received 2 courses of chemotherapy: docetaxel $35 \mathrm{mg} / \mathrm{m}^{2}$ with cisplatin $40 \mathrm{mg} / \mathrm{m}^{2}$ on days 1 and 15 and $400 \mathrm{mg} / \mathrm{m}^{2}$ 5-fluorouracil on days 1-5 and 15-19 every 4 weeks. After completion of the chemotherapy, patients received esophagectomy. The primary endpoint was the completion rate of protocol treatment. Thirty-two patients were enrolled. The completion rate of protocol treatment (completion of two courses of preoperative chemotherapy and R0 surgery) was $100 \%$. During chemotherapy, the most common grade 3 or 4 toxicities were neutropenia $(31.3 \%)$. No treatment-related death was observed, and the incidence of operative morbidity was tolerable. The overall response rate after the chemotherapy was $90.3 \%$. This Bi-DCF regimen was well tolerated and highly active. This trial was registered with the University Hospital Medical Information Network (No. UMIN 000014625).
\end{abstract}

Yoshihiro Tanaka

yoshihirotana11@hotmail.com

1 Department of Surgical Oncology, Gifu Graduate School of Medicine, 1-1 Yanagido, Gifu 501-1194, Japan

2 Department of Pathology, Gifu University Hospital, Gifu, Japan
Keywords Chemotherapy - Docetaxel · Cisplatin . 5-fluorouracil · Esophageal carcinoma

\section{Introduction}

Despite improvements in the modalities for early diagnosis of esophageal cancer, the majority of patients still tend to have widespread disease at the time of diagnosis [1]. Locally advanced esophageal carcinomas are treated with current modalities for early diagnosis and perioperative multimodalities. Although morbidity and mortality rates after surgical treatment for advanced esophageal cancer have decreased, the 5-year survival rate after curative surgery is still only $20-36 \%$ [2]. In patients with operable esophageal cancer, there is evidence supporting the use of preoperative chemotherapy [3, 4], but optimal chemotherapy for this disease has not been defined.

Patients with obvious T4 (non-resectable) or inoperable disease are usually treated with various chemotherapy strategies or chemoradiotherapy. However, their survival is still not satisfactory [5-8]. The most frequently used chemotherapy regimen for patients with metastatic disease is a combination of 5-fluorouracil (5-Fu) and cisplatin (CDDP), with response rates ranging from 15 to $45 \%$ [9].

Meanwhile, many chemotherapy regimens for locally advanced tumors have been reported to date. Despite the availability of various chemotherapy regimens, advanced esophageal cancer carries a very poor prognosis, with a mean survival time of less than 8.1 months with current chemotherapies used singly or in combination with 5-FU, vindesine, mitomycin, docetaxel (TXT), paclitaxel, CDDP, irinotecan, vinorelbine, or capecitabine [10]. Fluorouracil and CDDP combination therapy (FP) is regarded as standard [11], for which the median survival time is reported 
to be 9.2 months for responders and 5.3 months for nonresponders [12]. The response rates reported with FP range from 35 to $40 \%$, whereas the 2-year survival rates of patients with locally advanced esophageal cancer range from 8 to $55 \%$, with a mean of $27 \%$ [13-15].

The next generation of regimens to treat both distant metastasis and locally advanced cancer has begun to be developed. Many studies have shown that the taxanes have significant activity in patients with locally advanced and metastatic esophageal carcinomas [16-20].

The combination of TXT, CDDP, and 5-FU (DCF) has shown activity in upper gastrointestinal malignancies with different mechanisms. In Europe, DCF combination therapy is commonly used in patients with advanced gastric cancer. In a report from the V325 study group, patients with advanced gastric or gastroesophageal junction cancer receiving DCF not only had statistically improved overall survival and timeto-tumor progression, but they also had better preservation of quality of life compared with patients receiving FP therapy [21]. Advanced esophageal cancer might also benefit from the possibility of such a triple-combination regimen.

It is now necessary to reduce both the hematological and non-hematological toxicities as much as possible. Chemotherapy can significantly improve the clinical outcomes of cancer patients, but it can also cause serious adverse effects [22]. Treatment with DCF in esophageal cancer is reported to be associated with increased response rates but also with a highly increased incidence of toxicities, the most common of which are hematological and gastrointestinal [23, 24]. Thus, there is an urgent need for the creation of more effective treatment regimens with fewer adverse events. We therefore previously conducted a phase I clinical trial of DCF in patients with advanced thoracic esophageal squamous cell carcinoma (SCC) with T3-4 tumors and/or M1 staging with divided administration of each drug into two blocks per one course, i.e., biweekly DCF (Bi-DCF). We determined the recommended dose for use in phase II trials to be TXT $35 \mathrm{mg} / \mathrm{m}^{2}$, CDDP $40 \mathrm{mg} / \mathrm{m}^{2}$, and $5-\mathrm{Fu} 400 \mathrm{mg} / \mathrm{m}$, as was already reported [22]. In particular, preoperative chemotherapy for advanced esophageal carcinoma requires tolerability, because subsequent radical surgery for esophageal cancer is invasive for patients. We then carried out a phase II singlecenter study of preoperative chemotherapy with Bi-DCF in patients with clinical stage II/III thoracic esophageal SCC.

\section{Patients and methods}

\section{Patient eligibility criteria}

To be eligible for the study, patients had to be at least 18 years of age at the time of enrollment and have histologically or cytologically confirmed SCC, which was a locally advanced clinical stage II/III (International Union Against Cancer TNM classification system, 7th edition [25]). They also had to have an Eastern Cooperative Oncology Group (ECOG) performance status of $0-1$, a life expectancy of $>12$ weeks, and adequate liver, bone marrow, renal, and cardiovascular function as indicated by a serum bilirubin $\leq 1.5 \mathrm{mg} / \mathrm{dl}$, neutrophil count $\geq 1500 / \mathrm{mm}^{3}$, serum aspartate aminotransferase and alanine aminotransferase $\leq$ twice the upper limit of normal range, platelet count $\geq 10 \times 10^{4} /$ $\mathrm{mm}^{3}$, hemoglobin $\geq 8.0 \mathrm{~g} / \mathrm{dl}$, and creatinine $\leq 1.2 \mathrm{mg} /$ $\mathrm{dl}$ (or creatinine clearance $>60 \mathrm{ml} / \mathrm{min}$ ). Patients previously treated with chemotherapy for disease or irradiation to major bone areas were excluded from the study. The major exclusion criteria included serious concomitant illness, symptomatic infectious disease, severe drug allergy, symptomatic peripheral neuropathy, or uncontrolled diabetes mellitus.

\section{Study approval}

All participants had to sign an informed consent form approved by the Ethical Committee of Gifu University Hospital before study entry.

\section{Treatment plan}

\section{Chemotherapy}

Patients received TXT diluted in $250 \mathrm{ml}$ of normal saline at a dose of $35 \mathrm{mg} / \mathrm{m}^{2}$, which was infused intravenously (iv) over $2 \mathrm{~h}$, followed by a maintenance infusion given for the next $2 \mathrm{~h}$. Then, CDDP was prepared in normal saline at a dose of $40 \mathrm{mg} / \mathrm{m}^{2}$ and administered iv over $2 \mathrm{~h}$ on day 1 . 5 -FU was prepared in normal saline at a dose of $400 \mathrm{mg} /$ $\mathrm{m}^{2}$ and administered iv continuously on days $1-5$. TXT and CDDP were given on days 1 and 15, and 5-FU was given on days $1-5$ and $15-19$ of every 28 -day cycle (one course). All patients were premedicated with granisetron $2 \mathrm{mg}$ iv. Hypersensitivity reactions were treated with prophylactic use of dexamethasone $8 \mathrm{mg}$ iv, which was infused $1 \mathrm{~h}$ prior to the administration of TXT. Further, dexamethasone was prescribed at a dose of $4 \mathrm{mg}$ orally for 2 days after administration of the TXT to reduce the risk of hypersensitivity reaction and fluid retention. Diuretics were added at the discretion of the treating physician. Appropriate hydration was given before and after the CDDP infusion. Antiemetics were recommended on subsequent days as needed. The protocol did not allow the use of prophylactic granulocyte colony-stimulating factor (G-CSF) and antimicrobial therapy during chemotherapy. Patients were treated with hospitalization at first course. As long as serious adverse events did not occurred, all patients discharged at second course except days of drug administration. 


\section{Treatment assessment and dose modifications}

All patients underwent complete staging procedures to document disease extent, including ECOG performance status, medical history, and physical examination. Clinicopathological factors were analyzed based on the TNM classification [25].

Prestudy laboratory evaluation, including a complete blood cell count, serum electrolytes, urea, creatinine and 24-h creatinine clearance, bilirubin, alkaline phosphatase and transaminases, CEA, SCC, CA19-9, and CYFRA measurements, and electrocardiogram were obtained within 1 week before initiation of treatment and at the start of each treatment cycle. Baseline computed tomography (CT) or magnetic resonance imaging (MRI) scans were performed within 4 weeks prior to study entry. All patients had a complete blood count taken every week during chemotherapy. Levels of electrolytes, serum creatinine, transaminases, alkaline phosphatase and bilirubin, and plasma urea were measured every week until receiving surgery.

Toxicity was graded every week during the study according to the US National Cancer Institute-Common Terminology Criteria for Adverse Events (NCI-CTC) version 4.0 [26].

Measurable lesions except for the primary tumor were evaluated by CT or MRI and assessed according to the Response Evaluation Criteria in Solid Tumors (RECIST) [27]. A complete response was defined as complete disappearance of all clinically detectable malignant disease. A partial response was defined as a $\geq 30 \%$ decrease in the sum of the perpendicular diameters of all measurable lesions lasting at least 4 weeks. Progressive disease was defined as $a \geq 20 \%$ increase in the sum of the products of measurable lesions over the smallest sum observed, or the appearance of new lesions. Stable disease did not qualify as complete response, partial response, or progressive disease. Close follow-up was made from both endoscopy and radiographic films or scans taken to document treatment response during therapy and was repeated in the 4th week of every course of treatment and 4 weeks after completion of the 2 courses or sooner if the patient appeared to show disease progression.

Response rate (complete or partial) was confirmed 4 weeks after completion of the 2 courses of chemotherapy. Then, patients received right thoracotomy with thoracic esophagectomy within 14 days. A thoracoscopic surgery was permitted. If disease progression or new metastasis was detected after 1 course, the subsequent cycle was not permitted, and immediate surgery or chemoradiation was mandated. Regional lymphadenectomy consisted of two- or three-field extended lymphadenectomy. Evaluations of residual tumor $(\mathrm{R})$ were classified as follows: R0, no residual tumor; R1, suspicious of residual tumor or microscopic residual tumor; or R2, macroscopic residual tumor.

After surgery, the primary tumor was examined for histopathological changes using a grading system by the Japanese Classification of Esophageal Carcinoma [28], with the following grades: grade 3 (markedly effective; no viable cancer cells; pathologically complete response), grade 2 (moderately effective; viable cancer cells account for less than $1 / 3$ of tumor tissue, whereas other cancer cells show severe degeneration or necrosis), and grade 1 (slightly effective, where apparently viable cancer cells account for $1 / 3$ or more of the tumor tissue, but there is some evidence of degeneration of cancer tissue or cells). Grade 1 lesions were also subclassified into grade 1a (viable cancer cells account for $\geq 2 / 3$ of tumor tissue), grade $1 b$ (viable cancer cells account for $\geq 1 / 3$, but $<2 / 3$, of tumor tissue), and grade 0 (ineffective, denoting no discernible therapeutic effect on cancer tissue or cells).

Dose adjustment was carried out by reducing the doses of TXT, CDDP, and 5-Fu by $20 \%$ in the subsequent course if grade 4 hematological toxicity or grade 3 or 4 non-hematological toxicity was present. If there was no improvement in grade 3/4 toxicity on the day of predetermined course, we postponed the chemotherapy.

We terminated the protocol treatment if serious adverse reactions were manifested, clear progression of the disease was observed, or the physician otherwise judged that administration should be stopped.

\section{Endpoints and statistical methods}

The primary objectives of this phase II study was the compliance with treatment completion. Patients were considered to have completed treatment if they received 2 courses of chemotherapy and pathologically proven complete resection (R0). Secondary objectives included: the safety and tolerability of this chemotherapy; evaluation of operative morbidity and mortality; and evaluation of efficacy including response rate, pathological response, 1-year relapse-free survival (RFS), and overall survival (OS).

In this phase II trial, we expected that the clinical incidence of toxicities with Bi-DCF would increase above that with FP in the preoperative design, and that the rate of treatment completion would be lower than that in JCOG 9907 (89.6\%) [29]. Accordingly, we assumed a null hypothesis with a $75 \%$ completion rate for protocol treatment and expected a completion rate of protocol treatment of $90 \%$. Given a one-sided alpha of 0.1 and statistical power of $80 \%$, a minimum of 28 patients was needed. Assuming a dropout rate of $10 \%$, the projected sample size was 32 patients in total.

Relapse-free survival was defined as the time from the date of registration to the first documentation of disease 
recurrence. Overall survival was measured from the date of registration to the date of the last follow-up or death. Statistical data were obtained using the SPSS 20.0 software package (SPSS Inc., Chicago, IL, USA).

This trial was registered with the University Hospital Medical Information Network (No. UMIN 000014625).

\section{Results}

\section{Patient characteristics}

Between January 2010 and September 2014, 32 patients were enrolled into the study. Demographic and clinical characteristics of the study population are summarized in Table 1. All patients had locally advanced esophageal SCC. The median patient age was 68 years (range $40-75$ years). All patients had an ECOG performance status of $0-1$. Finally, 32 patients were enrolled, and all patients fully underwent Bi-DCF therapy.

Table 1 Characteristics of patients

\begin{tabular}{|c|c|c|}
\hline Characteristics & No. of patients $(N=32)$ & $\%$ \\
\hline \multicolumn{3}{|l|}{ Age, years } \\
\hline Median & 68 & \\
\hline Range & $40-75$ & \\
\hline \multicolumn{3}{|l|}{ Sex } \\
\hline Males & 26 & 81.3 \\
\hline Females & 6 & 18.8 \\
\hline \multicolumn{3}{|c|}{ ECOG performance status } \\
\hline $0-1$ & 32 & 100 \\
\hline 2 & 0 & 0 \\
\hline \multicolumn{3}{|c|}{ Site of primary tumor } \\
\hline Ut & 8 & 25.0 \\
\hline Mt & 15 & 46.9 \\
\hline $\mathrm{Lt}$ & 9 & 28.1 \\
\hline \multicolumn{3}{|l|}{ Clinical T stage } \\
\hline cT2 & 8 & 25.0 \\
\hline cT3 & 24 & 75.0 \\
\hline \multicolumn{3}{|l|}{ Clinical $N$ stage } \\
\hline $\mathrm{cNO}$ & 1 & 3.1 \\
\hline $\mathrm{cN} 1$ & 9 & 28.1 \\
\hline $\mathrm{cN} 2$ & 13 & 40.6 \\
\hline $\mathrm{cN} 3$ & 9 & 28.1 \\
\hline \multicolumn{3}{|l|}{ Clinical stage } \\
\hline IIB & 3 & 9.4 \\
\hline III & 29 & 90.6 \\
\hline
\end{tabular}

ECOG Eastern Cooperative Oncology Group, Ut upper thoracic esophagus, $M t$ middle thoracic esophagus, $L t$ lower thoracic esophagus

\section{Toxicity}

Overall toxicities during chemotherapy are listed in Table 2. The major toxicities were leukopenia and neutropenia. Two patients $(6.3 \%)$ had grade 4 and 8 patients (25\%) had grade 3 neutropenia; however, no patients had febrile neutropenia. Two patient with grade 4 neutropenia received G-CSF. Common non-hematological adverse events were anorexia, fatigue, mucositis, diarrhea, and alopecia. No grade 3 or 4 hyponatremia was occurred. All events were below grade 2 . No treatment-related deaths occurred. All toxicities were within expectations and were manageable.

\section{Surgery and postoperative complications}

All patients received surgery as listed in Table 3. Subtotal esophagectomy via right thoracotomy with two- or threefield lymphadenectomy was performed in 30 patients. Two patients received subtotal esophagectomy and lymphadenectomy via thoracoscopic surgery. We undergone reconstruction by stomach roll using subtotal stomach and hand-sewn anastomosis in cervical portion in all cases. All patients were considered to have achieved curative resection (R0).

Of the 32 patients who received surgery, postoperative complications (grade 2 or more according to NCI-CTCAE version 4.0) occurred in 3 patients in the form of grade 2 recurrent nerve palsy and in 1 patient in the form of grade 2 chylothorax. There were no anastomotic leakage and postoperative deaths.

\section{Treatment outcomes}

Of all 32 patients, no patients failed to complete 2 courses of chemotherapy and no patients required a delay of chemotherapy during any of the courses for adverse events. No patients required a dose reduction in the all courses because grade 4 neutropenia occurred after the completion of the 2 courses of the regimen.

All the patients received the surgery, and no patients required $\mathrm{R} 1$ resection pathologically. Thus, the completion rate (completion of 2 courses of preoperative chemotherapy and R0 surgery) of protocol treatment was $100 \%$.

Of the 31 patients who had measurable lesions, 6 $(19.4 \%)$ had a complete response and $22(71.0 \%)$ had a partial response to therapy, resulting in an overall response rate of $90.3 \%$ (95\% confidence interval $74.3-98.0 \%$ ) (Table 4).

The histological effects in the primary tumors were grade 3 in $7(21.9 \%)$ patients, grade 2 in $10(31.3 \%)$ patients, grade $1 \mathrm{~b}$ in $3(9.4 \%)$ patients, and grade $1 \mathrm{a}$ in 12 
Table 2 Frequency of treatment-related toxicity

\begin{tabular}{|c|c|c|c|c|c|c|}
\hline & \multicolumn{6}{|c|}{ CTCAE version 4.0 common toxicity criteria } \\
\hline & Grade 1 & Grade 2 & Grade 3 & Grade 4 & All grades $(\%)$ & Grade $3 / 4(\%)$ \\
\hline \multicolumn{7}{|l|}{ Hematological } \\
\hline Leucopenia & 5 & 12 & 4 & 0 & $21(65.6)$ & $4(12.5)$ \\
\hline Neutropenia & 2 & 7 & 8 & 2 & $19(59.4)$ & $10(31.3)$ \\
\hline Febrile neutropenia & - & - & 0 & 0 & 0 & 0 \\
\hline Anemia & 0 & 0 & 0 & 0 & 0 & 0 \\
\hline Thrombocytopenia & 0 & 1 & 0 & 0 & $1(3.1)$ & 0 \\
\hline \multicolumn{7}{|l|}{ Non-hematological } \\
\hline Anorexia & 8 & 5 & 0 & 0 & $13(40.6)$ & 0 \\
\hline Fatigue & 2 & 1 & 0 & 0 & $3(9.4)$ & 0 \\
\hline Mucositis & 2 & 9 & 0 & 0 & $11(34.4)$ & 0 \\
\hline Nausea/vomiting & 1 & 0 & 0 & 0 & $1(3.1)$ & 0 \\
\hline Diarrhea & 2 & 3 & 0 & 0 & $5(15.6)$ & 0 \\
\hline Pericardial effusion & - & 0 & 0 & 0 & 0 & 0 \\
\hline Alopecia & 23 & 9 & - & - & $32(100)$ & 0 \\
\hline Edema & 5 & 0 & 0 & 0 & $5(15.6)$ & 0 \\
\hline Sensory neuropathy & 0 & 0 & 0 & 0 & 0 & 0 \\
\hline Dysgeusia & 1 & 0 & 0 & 0 & $1(3.1)$ & 0 \\
\hline Hyponatremia & - & - & 0 & 0 & 0 & 0 \\
\hline
\end{tabular}

Data represent number of patients

CTCAE Common Terminology Criteria for Adverse Events of the National Cancer Institute
Table 3 Operative details and postoperative outcomes

\begin{tabular}{lll}
\hline & No of patients & $\%$ \\
\hline Surgical approach & 30 & 93.8 \\
Right thoracotomy & 2 & 6.3 \\
Thoracoscopic surgery & 32 & \\
Type of resection & 0 & 100 \\
R0 & & 0 \\
R1 & 3 & \\
Postoperative complications & 0 & 9.4 \\
Recurrent nerve palsy & 0 & 0 \\
Pneumonia & 0 & 0 \\
Anastomotic leakages & 0 & 0 \\
Pyothorax & 1 & 0 \\
Pneumothorax & 0 & 3.1 \\
Chylothorax & 0 & 0 \\
Wound infection & 0 & 0 \\
Heart failure & Postoperative mortality & 0 \\
\hline
\end{tabular}

$R O$ no residual tumor, $R I$ suspicious of residual tumor or microscopic residual tumor

(37.5\%) patients. The results also showed that $13(41.9 \%)$ of the 31 patients who were clinically diagnosed as positive for lymph node metastasis were pathologically node negative.
Table 4 Overall response in this phase II trial

\begin{tabular}{ll}
\hline & $N=31$ \\
\hline Complete response & $6(19.4 \%)$ \\
Partial response & $22(71.0 \%)$ \\
Stable disease & $3(9.7 \%)$ \\
Progressive disease & 0 \\
Overall response rate & $90.3 \%$ \\
Confidence interval & $74.3-98.0$ \\
\hline
\end{tabular}

The median follow-up period was 30 months (range 12-68 months). The 1-year RFS was $84.3 \%$. The 1-year survival rate was $90.6 \%$. The median RFS was not reached.

\section{Discussion}

Currently, additional treatments following surgery or radiotherapy are necessary to improve long-term patient outcome. In Western countries, neoadjuvant chemoradiotherapy is a standard treatment for locally advanced esophageal cancer [10]. Meanwhile, in Japan, neoadjuvant chemotherapy with FP has been approved as a standard regimen by the JCOG 9907 study [3]. There seemed to be a histological difference in the background of esophageal cancer, and although FP was an effective regimen, the response rate of 
$38 \%$ was unsatisfactory. Thus, a new regimen with low toxicity and high response is clearly desired.

In the present trial of Bi-DCF chemotherapy for esophageal SCC, a combination regimen with DCF was shown to be highly tolerable and effective in patients with clinical stage II/III cancer in preoperative setting.

The taxanes enhance polymerization of tubulin into stable microtubule formations and inhibit their tubulin depolymerization by blocking the cell cycle in metaphase, anaphase, and interphase [30]. This inhibition may improve the efficacy of drugs such as CDDP, which are active in all phases of the cell cycle via direct DNA damage. Furthermore, the taxanes increase programmed cell death, and TXT appears to be more potent than paclitaxel in inhibiting angiogenesis [31].

TXT, CDDP, and 5-FU activity occurs by synergistic or non-cross-resistance effects when administered in combination [24]. Previously published studies have shown that the DCF combination has good efficacy [23, 32-38]. A $34.5-83.3 \%$ response rate was observed with DCF combinations used to treat patients with advanced esophageal carcinoma (Table 5).

We found that our Bi-DCF regimen also had a high response rate and showed highly promising antitumor activity. The histological grade $2 / 3$ rate was $53.2 \%$, which is high compared with the $25-51 \%$ reported previously $[23,36,37]$. The results emerging from this phase II study are particularly encouraging. By dividing the single dose of each of these three powerful drugs, it is not necessary to reduce their doses in the second course. Because this may eventually increase the dose intensity of the antitumor agent, it is likely that a high response rate can be obtained.

Previous studies indicated that this triplet regimen seems to be not inferior to chemoradiotherapy with respect to the local control rate [23, 39-41]. In the present study, $24(75.0 \%)$ patients were clinically $\mathrm{T} 3$; remarkably, the histological effects regarding T3 tumor were grade 3 in $7(29.2 \%)$, grade 2 in $8(33.3 \%)$, grade $\mathrm{Ib}$ in $1(4.2 \%)$, and grade Ia in $8(33.3 \%)$ patients. Therefore, this regimen might lead to more cases of locally highly advanced esophageal SCC.

Although TXT offers favorable outcomes, it causes adverse hematological toxicity. Because of high rate of blood toxicity resulting from the administration method used in previous reports, there appeared to be difficulties in enforcing the regimen in general hospitals. Neutropenia occurs approximately 8-10 days after TXT administration but recovers rapidly. TXT $75-100 \mathrm{mg} / \mathrm{m}^{2}$ every $3-4$ weeks is associated with a quite pronounced neutropenia, up to $44 \%$ rate of febrile neutropenia in patients with recurrent ovarian cancer [42]. Several reports revealed that the major toxicity of DCF repeated every 3-4 weeks at doses of TXT
$50-75 \mathrm{mg} / \mathrm{m}^{2}$, CDDP60-75 mg/m ${ }^{2}$, and 5-FU 700-800 mg/ $\mathrm{m}^{2}$ was myelosuppression, and the frequencies of grade $3 / 4$ leucopenia and neutropenia in a phase II study were $33.3 \%$ and $90 \%$, respectively [23, 32-36], (Table 5). In our biweekly regimen, the incidence of hematological toxicity was lower than that in the other reports. Although we did not use a prophylactic antimicrobial agent, no febrile neutropenia occurred, and no thrombocytopenia $\geq$ grade 3 was observed.

To minimize toxicity and maximize the total dose intensity, we elected to investigate a biweekly regimen. Compared with the other DCF regimens for esophageal cancer of other phase I/II studies [23, 32-38], (Table 5), our regimen included a TXT dose intensity equivalent to the triweekly administration of TXT performed in those studies. Divided administration of TXT and CDDP may reduce myelosuppression and neuropathy while maintaining almost unchanged efficacy. Tebbutt et al. [24] showed that weekly TXT administration in the triplet DCF regimen reduced blood toxicity for patients with esophagogastric cancer.

In the present phase II trial, the incidence of TXT-specific toxicities, such as neurotoxicity and acute hypersensitivity reactions [22], was relatively low and did not appear to be a major clinical problem, so a reduction in dose generally was not required. This was probably due to the low per-day dose of TXT administered via the biweekly method. Fluid retention manifesting as peripheral edema, pleural effusion, or ascites was cumulative in incidence and severity, but no patient had severe body weight gain that required diuretics. Patients receiving more than $50 \mathrm{mg} / \mathrm{m}^{2}$ of CDDP may suffer nausea and vomiting [43]. Both are frequent side effects and can be well controlled by biweekly method and the administration of granisetron and dexamethasone. Thus, nonhematological toxicity was comparable to that of other reports.

The Bi-DCF regimen seemed to be useful as preoperative chemotherapy due to the small need to delay the anticancer drug administration schedule, and it did not adversely affect our elective surgery schedule. In fact, surgery was carried out according to plan in all 32 patients, and no surgeries were delayed. Moreover, there was no mortality, and no serious postoperative complications occurred.

In conclusion, the preoperative Bi-DCF regimen was well tolerated and useful for the treatment of resectable esophageal SCC. It could potentially be offered as a candidate component of standard regimens for treating resectable esophageal SCC. A further phase III clinical trial using this triplet combination should be pursued in the treatment of advanced esophageal SCC. 


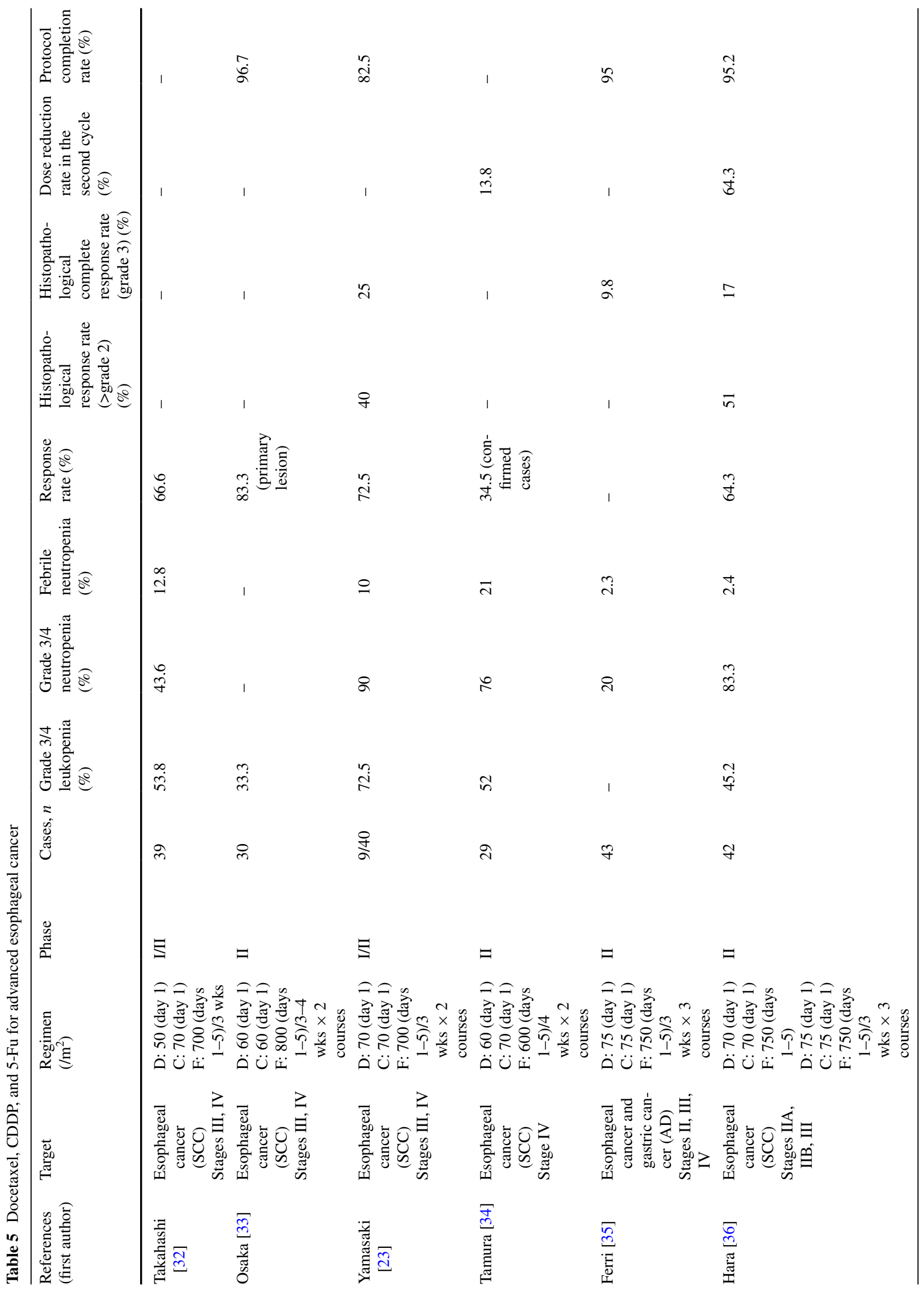




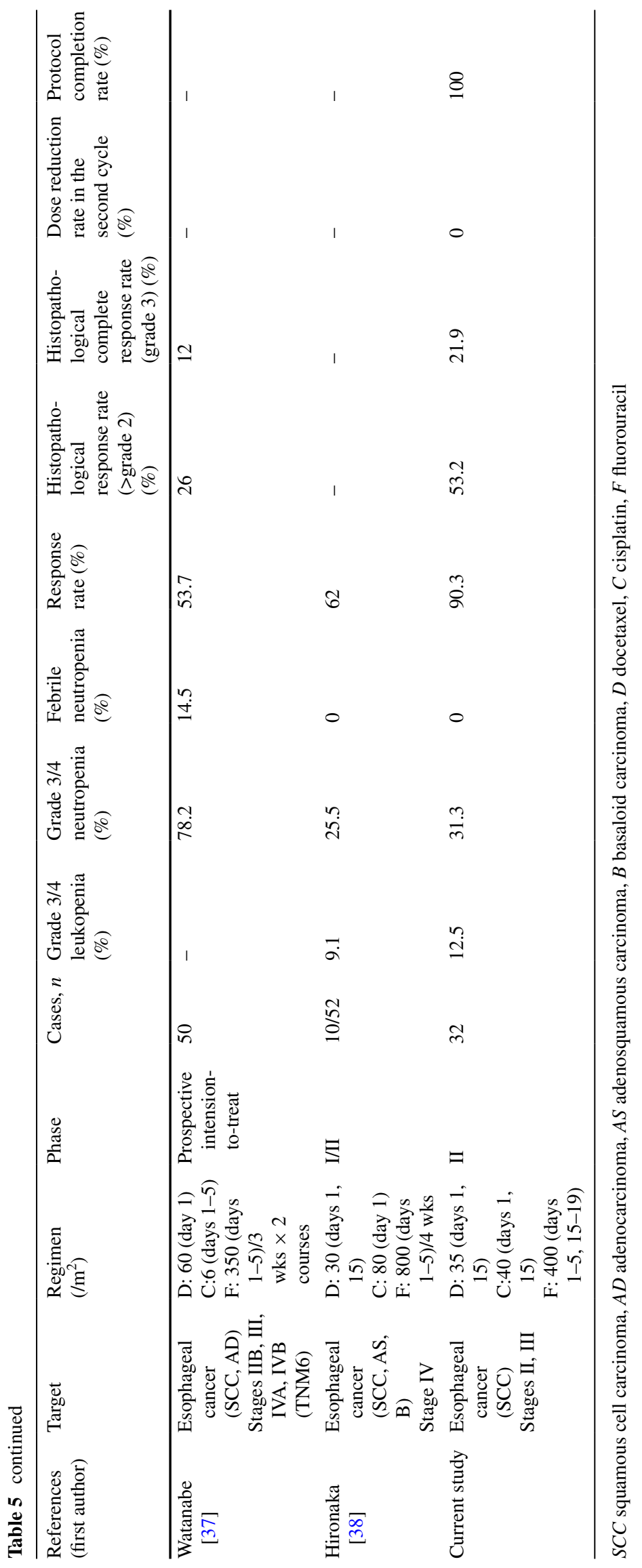

Springer 
Acknowledgments We would like to thank Rise Japan LLC for providing editorial assistance.

\section{Compliance with ethical standards}

Conflict of interest The authors declare that they have no competing interests.

Open Access This article is distributed under the terms of the Creative Commons Attribution 4.0 International License (http://creativecommons.org/licenses/by/4.0/), which permits unrestricted use, distribution, and reproduction in any medium, provided you give appropriate credit to the original author(s) and the source, provide a link to the Creative Commons license, and indicate if changes were made.

\section{References}

1. Morita M, Yoshida R, Ikeda K et al (2008) Advances in esophageal cancer surgery in Japan: an analysis of 1000 consecutive patients treated at a single institute. Surgery 143:499-508

2. Bagheri R, Maddah G, Saedi HS, Sadeghian MH, Roodbari S (2011) Bone marrow involvement in esophageal cancer patients who underwent surgical resection. Eur J Cardiothorac Surg 40:343-346

3. Ando N (2011) Progress in multidisciplinary treatment for esophageal cancer in Japan as reflected in JCOG studies. Esophagus 8:151-157

4. Khaldoun A, Jonathan RS (2012) Multimodality approach for locally advanced esophageal cancer. World J Gastroenterol 18:5679-5687

5. Coonley CJ, Bains M, Heelan R, Dukeman M, Kelsen DP (1983) Phase II study of etoposide in the treatment of esophageal carcinoma. Cancer Treat Rep 67:397-398

6. Crosby TD, Brewster AE, Borley A et al (2004) Definitive chemoradiation on patients with inoperable oesophageal carcinoma. Br J Cancer 90:70-75

7. Bedenne L, Michel P, Bouché O et al (2007) Chemoradiation followed by surgery compared with chemoradiation alone in squamous cancer of the esophagus: FFCD 9102. J Clin Oncol 25:1160-1168

8. Kranzfelder M, Buchler P, Lange K, Friess H (2010) Treatment options for squamous cell cancer of the esophagus: a systematic review of the literature. J Am Coll Surg 210:351-359

9. Ilson DH (2003) Oesophageal cancer: new developments in systemic therapy. Cancer Treat Rev 29:525-532

10. Chiarion-Sileni V, Corti L, Ruol A et al (2007) Phase II trial of docetaxel, cisplatin and fluorouracil followed by carboplatin and radiotherapy in locally advanced oesophageal cancer. Br J Cancer 96:432-438

11. Cooper JS, Guo MD, Herskovic A et al (1999) Chemoradiotherapy of locally advanced esophageal cancer: long-term follow-up of a prospective randomized trial (RTOG 85-01) Radiation Therapy Oncology Group. JAMA 281:1623-1627

12. Iizuka T, Kakegawa T, Ide H et al (1992) Phase II evaluation of cisplatin and 5-fluorouracil in advanced squamous cell carcinoma of the esophagus: a Japanese Esophageal Oncology Group Trial. Jpn J Clin Oncol 22:172-176

13. De Besi P, Sileni VC, Salvagno L et al (1986) Phase II study of cisplatin, 5-FU, and allopurinol in advanced esophageal cancer. Cancer Treat Rep 70:909-910

14. Bleiberg H, Conloy T, Paillot B et al (1997) Randomised phase II study of cisplatin and 5-fluorouracil (5-FU) versus cisplatin alone in advanced squamous cell oesophageal cancer. Eur J Cancer 33:1216-1220

15. Honda M, Miura A, Izumi Y et al (2010) Doxorubicin, cisplatin and fluorouracil combination therapy for metastatic esophageal squamous cell carcinoma. Dis Esophagus 23:641-645

16. Rigas JR, Dragnev KH, Budis JA (2005) Docetaxel in the treatment of esophageal cancer. Semin Oncol 32:S39-S51

17. Yoshida K, Ninomiya M, Takakura N et al (2006) Phase II study of docetaxel and S-1 combination therapy for advanced or recurrent gastric cancer. Clin Cancer Res 12(11 Pt 1):3402-3407

18. Wada Y, Yoshida K, Suzuki T et al (2006) Synergistic effects of docetaxel and S-1 by modulating the expression of metabolic enzymes of 5-fluorouracil in human gastric cancer cell lines. Int J Cancer 119:783-791

19. Hihara J, Yoshida K, Hamai Y, Emi M, Yamaguchi Y, Wadasaki K (2007) Phase I study of docetaxel (TXT) and 5-fluorouracil (5-FU) with concurrent radiotherapy in patients with advanced esophageal cancer. Anticancer Res 27:2597-2603

20. Tanaka Y, Yoshida K, Osada S, Yamaguchi K, Takahashi T (2011) Docetaxel, nedaplatin, and S-1 (DGS) chemotherapy for advanced esophageal carcinoma: a phase I dose-escalation study. Anticancer Res 31:4589-4598

21. Ajani JA, Moiseyenko VM, Tjulandin S et al (2007) Quality of life with docetaxel plus cisplatin and fluorouracil compared with cisplatin and fluorouracil from a phase III trial for advanced gastric or gastroesophageal adenocarcinoma: the V-325 Study Group. J Clin Oncol 25:3210-3216

22. Tanaka Y, Yoshida K, Sanada Y, Osada S, Yamaguchi K, Takahashi T (2010) Biweekly docetaxel, cisplatin, and 5-fluorouracil (DCF) chemotherapy for advanced esophageal squamous cell carcinoma: a phase I dose-escalation study. Cancer Chemother Pharmacol 66:1159-1165

23. Yamasaki M, Miyata H, Tanaka K et al (2011) Multicenter phase I/II study of docetaxel, cisplatin and fluorouracil combination chemotherapy in patients with advanced or recurrent squamous cell carcinoma of the esophagus. Oncology 80:307-313

24. Tebbutt NC, Cummins MM, Sourjina T et al (2010) Randomized, non-comparative phase II study of weekly docetaxel with cisplatin and 5-fluorouracil or with capecitabine in oesophagogastric cancer: the AGITG ATTAX trial. Br J Cancer 102:475-481

25. International Union Against Cancer (UICC) (2009) TNM Classification of Malignant tumors. In: Sobin LH, Wittekind C (eds) Wiley, New York

26. National Cancer Institute: National Cancer Institute Common Terminology Criteria for Adverse Events v 4.0: May 28, 2009

27. Therasse P, Arbuck SG, Eisenhauer EA et al (2000) New guidelines to evaluate the response to treatment in solid tumors. European Organization for Research and Treatment of Cancer, National Cancer Institute of the United States, National Cancer Institute of Canada. J Natl Cancer Inst 92:205-216

28. Japan Esophageal Society (2009) Japanese classification of esophageal cancer, tenth edition: part II and III. Esophagus 6:71-94

29. Ando N, Kato H, Igaki H et al (2012) A randomized trial comparing postoperative adjuvant chemotherapy with cisplatin and 5-fluorouracil versus preoperative chemotherapy for localized advanced squamous cell carcinoma of the thoracic esophagus (JCOG9907). Ann Surg Oncol 19:68-74

30. Schiff PB, Fant J, Horwitz SB (1979) Promotion of microtubule assembly in vitro by taxol. Nature 277:665-667

31. Grant DS, Williams TL, Zahaczewsky M, Dicker AP (2003) Comparison of antiangiogenic activities using paclitaxel (taxol) and docetaxel (taxotere). Int J Cancer 104:121-129

32. Takahashi H, Arimura Y, Yamashita K et al (2010) Phase I/ II study of docetaxel/cisplatin/fluorouracil combination 
chemotherapy against metastatic esophageal squamous cell carcinoma. J Thorac Oncol 5:122-128

33. Osaka Y, Shinohara M, Hoshino S et al (2011) Phase II study of combined chemotherapy with docetaxel, CDDP and 5-FU for highly advanced esophageal cancer. Anticancer Res 31:633-638

34. Tamura S, Imano M, Takiuchi H et al (2012) Phase II study of docetaxel, cisplatin and 5-fluorouracil (DCF) for metastatic esophageal cancer (OGSG 0403). Anticancer Res 32:1403-1408

35. Ferri LE, Ades S, Alcindor T et al (2012) Perioperative docetaxel, cisplatin, and 5-fluorouracil (DCF) for locally advanced esophageal and gastric adenocarcinoma: a multicenter phase II trial. Ann Oncol 23:1512-1517

36. Hara H, Tahara M, Daiko H et al (2013) Phase II feasibility study of preoperative chemotherapy with docetaxel, cisplatin, and fluorouracil for esophageal squamous cell carcinoma. Cancer Sci 104:1455-1460

37. Watanabe M, Baba Y, Yoshida N et al (2014) Outcomes of preoperative chemotherapy with docetaxel, cisplatin, and 5-fluorouracil followed by esophagectomy in patients with resectable nodepositive esophageal cancer. Ann Surg Oncol 21:2838-2844

38. Hironaka S, Tsubosa Y, Mizusawa J et al (2014) Phase I/II trial of 2-weekly docetaxel combined with cisplatin plus fluorouracil in metastatic esophageal cancer (JCOG0807). Cancer Sci 105:1189-1195
39. Ikeda K, Ishida K, Sato $\mathrm{N}$ et al (2001) Chemoradiotherapy followed by surgery for thoracic esophageal cancer potentially or actually involving adjacent organs. Dis Esophagus 14:197-201

40. Ishida K, Ando N, Yamamoto S, Ide H, Shinoda M (2004) Phase II study of cisplatin and 5-fluorouracil with concurrent radiotherapy in advanced squamous cell carcinoma of the esophagus: a Japan Esophageal Oncology Group (JEOG)/Japan Clinical Oncology Group trial (JCOG9516). Jpn J Clin Oncol 34:615-619

41. Fujita H, Sueyoshi S, Tanaka T et al (2005) Prospective non-randomized trial comparing esophagectomy-followed-by-chemoradiotherapy versus chemoradiotherapy-followed-by-esophagectomy for T4 esophageal cancers. J Surg Oncol 90:209-219

42. Kaye SB, Piccart M, Aapro M, Francis P, Kavanagh J (1997) Phase II trials of docetaxel (Taxotere) in advanced ovarian cancer-an updated overview. Eur J Cancer 33:2167-2170

43. Gralla RJ, Osoba D, Kris MG et al (1999) Recommendations for the use of antiemetics: evidence-based, clinical practice guidelines. American Society of Clinical Oncology. J Clin Oncol 17:2971-2994 